\title{
Traffic States Detection based on Block Matching
}

\author{
Qinglin Jiang \\ Guizhou Minzu University \\ Guiyang, China \\ Zaijun Zhang \\ Guizhou Minzu University
}

\author{
Lin Wang \\ Guizhou Minzu University \\ Guiyang, China \\ wanglin@gzmu.edu.cn
}

\begin{abstract}
A traffic states detection method is proposed to represent the road traffic conditions accurately and reduce labor intensive monitoring work in this paper. The edge of image, which is named as edge characteristic, is applied to describe the number of vehicles running on the road, and the average density of vehicle edge pixels is used to substitute the road occupation ratio. Meanwhile block matching method is used for estimating the speed of traffic flow. According the extracted feature parameters, a classifier is designed to classify the traffic states in three categories (congested, slow, and smooth). The experimental results showed the proposed algorithm is robust.
\end{abstract}

Keywords-Traffic state detection; Traffic flow speed; Block matching; Vehicle edge density; Background subtract

\section{INTRODUCTION}

Traffic congestion problem has become a global issue which impacts the urban normal functioning and sustainable development. Undoubtedly, it is necessary to build a video-based traffic surveillance system which can provide accurate and real-time traffic information anywhere and anytime. Most existing traffic state detection methods based on image segmentation and vehicle tracking, however, one of its disadvantages is that accuracy depends on the quality of image segmentation and the segmentation task is easily influenced by such as lighting, shadow and occlusion so that target tracking becomes more difficult. In fact, traffic states (such as congestion, slow, smooth, accident) are macroscopic description of road' s traffic flow. Tan apply edge texture feature in ROI to classify traffic flow density into four levels (empty, low, high and full $)^{[1]}$. Bi proposed an algorithm of Gaussian group-based histogram (GBH) to build background image, and describe the amount of vehicle running on the road according to average intensity of background subtract image $^{[2]}$. Li proposed a traffic state detection system which can estimate traffic flow speed and road space occupancy, and recognize three typical traffic states (congested, slow, and smooth) ${ }^{[3]}$. In his paper, using the feature optical flow vectors to calculate MOFV (macroscopic optical flow velocity), and applying the MOFV values of each main direction to estimate traffic flow speed of corresponding direction. Moreover, the road areas can be extracted by motion accumulation based on frame difference, however, the approach of frame difference will be interfered easily by the illumination changes and sign of trouble result in failing to detect the road areas. Li proposed a traffic state detection system which can estimate traffic flow speed and road space occupancy, and recognize three typical traffic states (congested, slow, and smooth) ${ }^{[4]}$. In his paper, using the feature optical flow vectors to calculate MOFV, and applying the MOFV values of each main direction to estimate traffic flow speed of corresponding direction. Moreover, the road areas can be extracted by motion accumulation based on frame difference, however, the approach of frame difference will be interfered easily by the illumination changes and sign of trouble result in failing to detect the road areas. Ouessai Asmaa estimated road traffic density using microscopic and macroscopic parameters, and classified road traffic congestion in light, medium and heavy ${ }^{[5]}$. In traffic state detection, these motion analysis based methods can achieve a higher matching ratio with the visual processing of human beings, thereby it is unnecessary for individual vehicle detection, meanwhile enhance classification accuracy and reduce computational complexity.

In this paper ROI (region of interest) set up is required in advance, using Gaussian Mixture Model (GMM) to create background. Applying edge pixel density on road areas to represent the road traffic occupancy, meanwhile, block matching technique is used for estimating the mean velocity of road traffic flow. Finally, three typical traffic states (congestion, slow, and smooth) can be classified by the classifier which is achieved using feature parameters, extracted from the video stream.

\section{TRAFFIC STATE DETECTION METHOD OVERVIEW}

Figure 1. shows the process of traffic state detection system. The road background is calculated from the image sequence by Gaussian Mixture Model (GMM). The road occupation ratio is estimated using image edge features extraction on road areas. Block matching is used to estimate the block motion vector, the mean velocity of motion vectors, which is named as speed characteristic, is used to characterize the speed of the traffic flow. And the traffic state detection algorithm is presented with those two features, image edge characteristic and speed characteristic finally. 


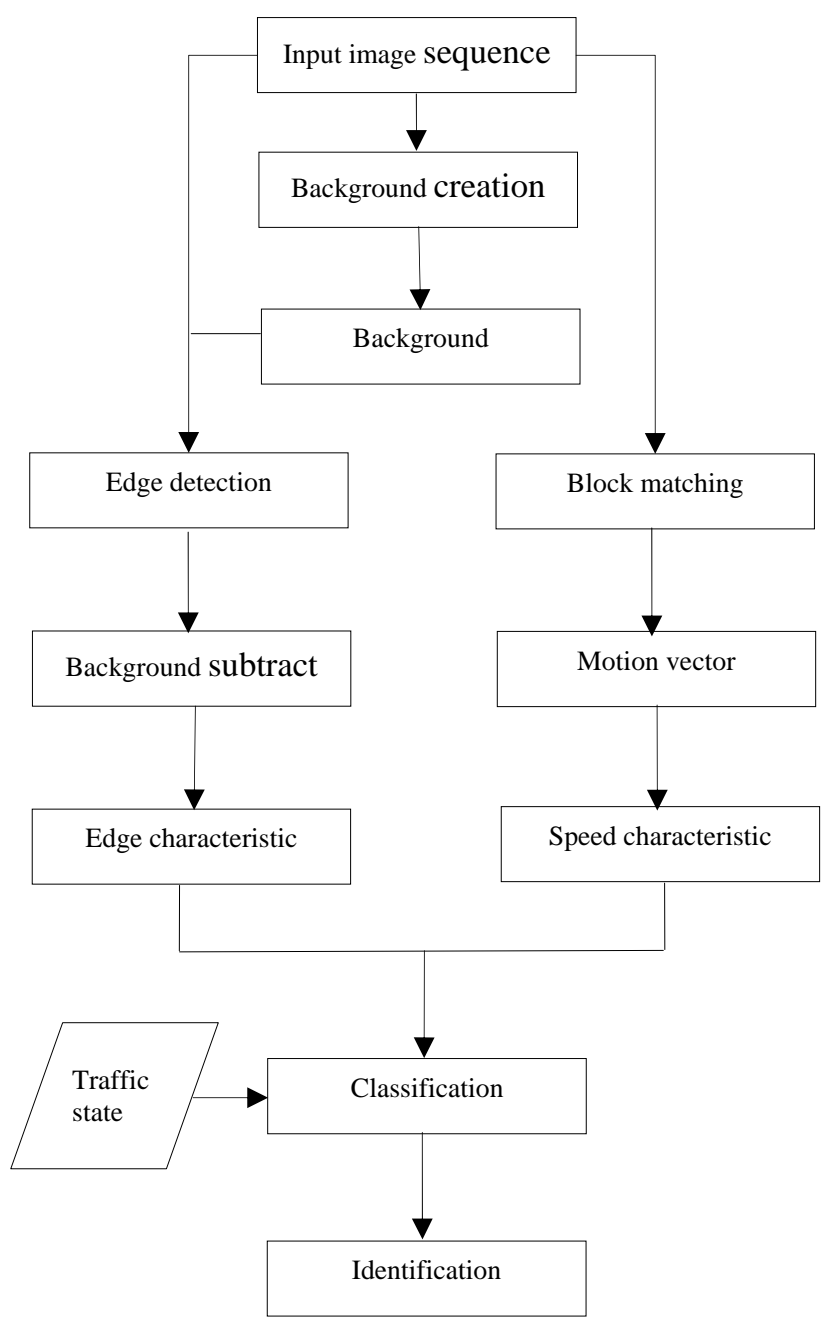

Figure 1. Flow chart of traffic state detection system

\section{CHARACTERISTIC PARAMETERS EXTRACTION}

\section{A. Image edge characteristic extraction}

\section{1) Background creation}

Road areas extraction is useful to gain calibration parameters and reduce the calculation work which is the basis of road occupancy calculation. Li accumulated road areas by a consecutive sequence of binary frame difference image accumulation, and employ Hough detect the road structure. However, the accuracy of road areas accumulation is interfered by camera shaking and illumination changes. In this paper, we initialize area we are interested in, and creating road background image by Gaussian Mixture Model (GMM), the created background is shown in Figure 2.

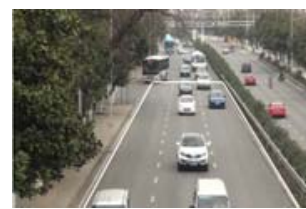

(a)Region of interest

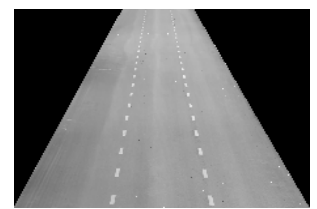

(b) background
Figure 2. The results of background creation

Figure 3.
2) Edge characteristic extraction

Using Sobel algorithm to detect background and current frame edge, the edge map is extracted by background difference image which is acquired by the union operator of edge image.

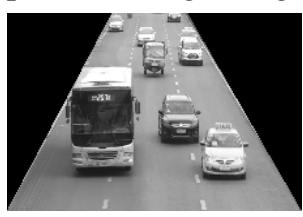

(a) current frame

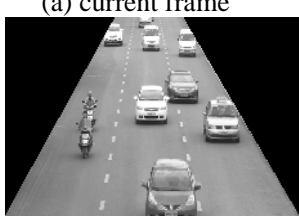

(d)current frame

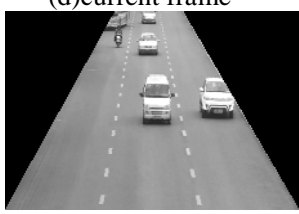

(f) current frame

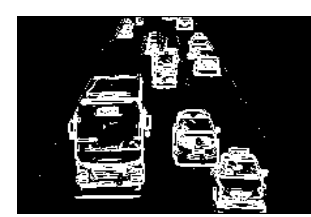

(b) edge extraction

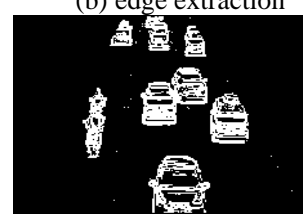

(e)edge extraction

(g) edge extraction

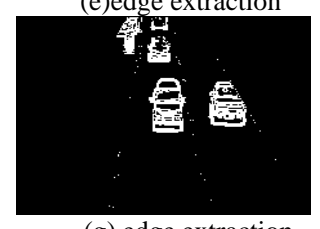

Figure 4. The results of edge characteristic extraction

\section{3) Edge density calculation}

In fact, there is a linear correlative relationship between the average intensity of background subtract image the occupation ratio ${ }^{[6-8]}$.

Let $g$ denote the image of current frame, $g_{B}$ denote the image of background. The background subtracted image is calculated by $g_{D}=\left|g-g_{B}\right|$.

Let $N$ denote the pixel amount of road area in observation area, $N_{c}$ denotes the pixel amount of vehicle, Assuming $R_{o}=\frac{N_{C}}{N}$ is the road occupancy rate.

Let $C_{r}$ denote a bounded set of pixel the which belonging to the road in the ROI, the average intensity of background is $\bar{g}_{B}$, the average intensity of vehicle area is $\bar{g}_{C}$.The average intensity of subtracted image $\bar{g}_{D}$ is calculated by following equation.

$$
\begin{gathered}
\left.\bar{g}_{D}=1 / N \cdot\left[\left(N-N_{C}\right) \bar{g}_{B}+N_{C} \bar{g}_{C}\right)\right]= \\
1 / N\left[\bar{N}_{B}+N_{C}\left(\bar{g}_{C}-\bar{g}_{B}\right)\right]=\bar{g}_{B}+N_{C} / N_{N}\left(\bar{g}_{C}-\bar{g}_{B}\right) \\
\bar{g}_{B}=1 / P Q \sum_{j=1}^{P} \sum_{i=1}^{Q}\left|g(i, j)-g_{B}(i, j)\right|
\end{gathered}
$$

In (2), $g(i, j) \in C_{r}, g_{B}(i, j) \in C_{r}, P$ and $Q$ denote the width and height of the frame respectively. As background is the estimation to the road area, $\left|g(i, j)-g_{B}(i, j)\right| \leq \varepsilon$ for the pixel in set $C_{r}$ and $\varepsilon$ is a small infinitely small quantity. The following express we can get according to (1) and (2) . 


$$
\begin{gathered}
\bar{g}_{B} \leq 1 / P Q \sum_{j=1}^{P} \sum_{i=1}^{Q} \varepsilon=\varepsilon \\
\bar{g}_{D}=\bar{g}_{B}+N_{C} / N\left(\bar{g}_{C}-\bar{g}_{B}\right) \leq \varepsilon+R_{o}\left(\bar{g}_{C}-\varepsilon\right) \\
\bar{g}_{D} \approx R_{o} \bar{g}_{C}(\varepsilon \rightarrow 0)
\end{gathered}
$$

The value region of $\bar{g}_{D}$ is in the range of $[0,1]$, and bigger value means higher pixels' density of vehicle on road areas.

As edge is a kind of robust image feature with few changes while illumination is changing, the vehicle density on road is high, the density of vehicle's feature on road also reaches a high level. The density of vehicle can be substituted by the density of image features when it is difficult to distinguish the individual vehicle in surveillance video, so we can use edge pixels' density on road areas represents the road traffic occupancy. The value of edge pixels' density is expressed as:

$$
O=\frac{N_{E}}{N}
$$

Where $N_{E}$ is the pixel amount of vehicle edge.

\section{B. Speed characteristic extraction}

\section{1) Block matching method}

Block matching technique is commonly used for estimating the block motion vector due to its simplicity ${ }^{[9-}$ 11].

The matching error between the block at position $(x, y)$ in the current image, and the candidate block at position $(x+u, y+v)$ in the reference image, The Sum of Absolute Difference (SAD) is often recapped by equation as follows.

$$
\begin{aligned}
& S A D_{(x, y)}(u, v)=\sum_{i-0}^{B-1} \sum_{j=0}^{B-1} \mid I_{t}(x+i, y+j)- \\
& I_{t-1}(x+u+i, y+v+j) \mid
\end{aligned}
$$

Where $\mathrm{B}$ is the block size.

The best estimate of the block motion vector is $(\hat{u}, \hat{v})$ the one having the minimum matching error:

$$
\left(\hat{u, v)}=\arg \min _{(u, v)} S A D_{(x, y)}(u, v)\right.
$$

We used a search window size $(2 \mathrm{R} \times 2 \mathrm{R})$ for blocks of size $(R \times R)$, where $R=16$ pixels.

Road traffic velocity is estimated by the mean velocity of the non-zero motion vectors (the amount is $n$ ).The length of a motion vector is defined as the moving distance of the corresponding block which is given by following equation:

$$
V=\frac{1}{n} \sum_{n} \max \left(\sqrt{\hat{u}^{2}+\hat{v}^{2}}\right)
$$

\section{CLASSIFICATION OF TRAFFIC STATES}

\section{A. Classifier design}

Through the analysis of road traffic flow in the video scene, it is easy to find the facts: if the amount of vehicle running on the road is small, traffic flow speed is large, and road space occupancy is also low, the traffic state is smooth usually, if vehicle is more and its speed is slower even zero and road space occupancy is high, the traffic state is congested, what between smooth state and congestion state is slow state. So we design a classifier based on the value of speed characteristic and edge characteristic threshold segment, the thresholds of speed and edge density, $T_{V}$ and $T_{E}$, are set according to the video scene and experience value. The structure of traffic state classifier is shown in Figure 4.

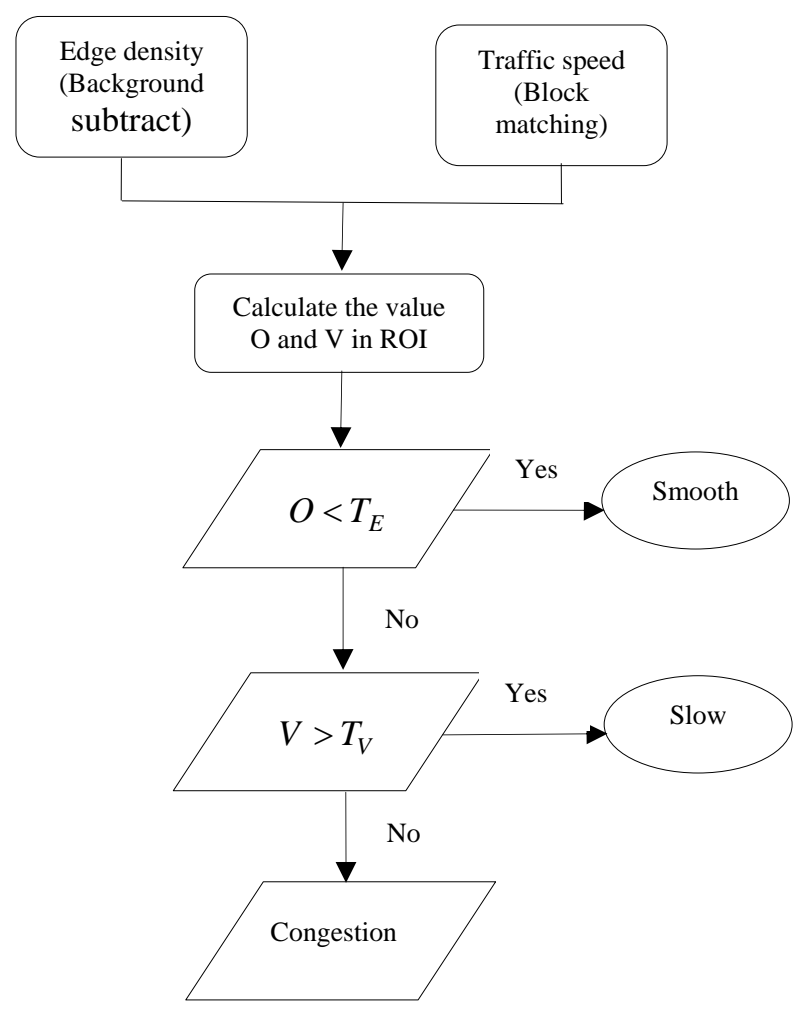

Figure 5. The structure of traffic state classifier

\section{EXPERIMENTAL RESULTS}

The surveillance videos which is taken on May 10, 2015 in Guiyang Huaxi , are used to test the validity of the above algorithm. The system software is developed by Matlab7.1 or Visual C++ 6.0 and Windows XP system. The basic hardware configuration is Pentium3.39GHz CPU with 4G memories. The process of background build, feature parameters extraction and traffic state classification are cost $30.2 \mathrm{~ms}$. The surveillance videos include different scenes and some are shown in Figure 5. The parameters and classification results are listed in Table 1 . Here, edge density threshold $T_{E}$ is 0.4 , and speed with block matching threshold $T_{V}$ is 0.7 pixels/frame. 


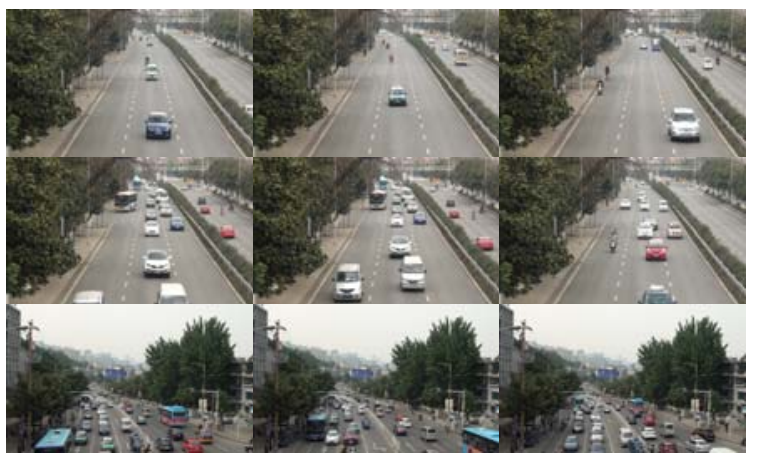

Figure 6. Example of frames from the traffic video data set. The sample frames depict various traffic conditions, coarsely categorized as smooth (top row), slow (middle row) and congested (bottom row).

TABLE I. THE RESULTS OF SAMPLES TESTING

\begin{tabular}{ccccc}
\hline & \multicolumn{2}{c}{ Average value } & \multicolumn{2}{c}{ Classification results } \\
\cline { 2 - 5 } Number & Edge density & Speed & $\begin{array}{c}\text { Proposed } \\
\text { method }\end{array}$ & $\begin{array}{c}\text { Real traffic } \\
\text { states }\end{array}$ \\
\hline 1 & 0.15 & 0.86 & Smooth & Smooth \\
2 & 0.12 & 0.90 & Smooth & Smooth \\
3 & 0.14 & 0.88 & Smooth & Smooth \\
4 & 0.42 & 0.75 & Slow & Slow \\
5 & 0.43 & 0.70 & Slow & Slow \\
6 & 0.45 & 0.67 & Slow & Slow \\
7 & 0.77 & 0.53 & Congested & Congested \\
8 & 0.74 & 0.49 & Congested & Congested \\
\hline 9 & 0.78 & 0.37 & Congested & Congested \\
\hline
\end{tabular}

In order to test effectiveness of classifier, another 1000 samples are extracted from video flow, among them, 98 congestion sections were detected correctly. The main reason is that background model does not have the flexibility to long stop of vehicles.

\section{CONCLUSIONS}

A new method to detect traffic states in this paper is presented. The proposed method is based on block matching used for estimating traffic flow velocity and image edge characteristic extracted by background subtract. The best performance was obtained by using classifier with the mean speed of traffic and edge density. The test results have shown that the proposed algorithm successfully detect traffic states with a good robustness.

\section{ACKNOWLEDGMENT}

This work was supported by National Natural Science Foundation of China (61263034) and Natural Science Foundation of Guiyang.

\section{REFERENCES}

[1] E. Tan, J. Chen, "Vehicular Traffic Density Estimation via Statistical Methods with Automated State Learning,” In Processing of IEEE Conference on Advanced Video and Signal Based Surveillance, pp. 164-169, 2007.

[2] Song Bi, Liqun Han, Yixin Zhong, Xiaojie Wang, “All-day traffic states recognition system without vehicle segmentation,” The Journal of China Universities of Pots and Telecommunications. Vol. 18, pp. 1-11, 2011.

[3] Xiying Li, Yongye She, Guigen Yang, Youting Zhao, Donghua Luo, "A Traffic Congestion Detection Method for Surveillance Videos Based on Macro Optical Flow Velocity," In The 11th International Conference of Chinese Transportation Professionals (ICCTP11), pp.1569-1578, 2011.

[4] Xiying Li, Yongye She, Donghua Luo, Zhi Yu, "A Traffic State Detection Tool for Freeway Video Surveillance System," 13th COTA International Conference of Transportation Professionals (CICTP13), Procedia Social and Behavioral Sciences, vol.96, pp.2453-2461, 2013.

[5] Ouessai Asmaa, Keche Mokhtar, Ouamri Abdelaziz, "Road traffic density estimation using microscopic and macroscopic parameters,” IEEE Trans. Image and Vision Computing, pp. 887894, 2013.

[6] Benjamin Coifman, David Beymer, Philip McLauchlan, Jitendra Malik, "A real-time computer vision system for vehicle tracking and traffic surveillance,” Transportation Research Part C, Vol. 6, pp. 271-288.2008.

[7] Fu Yuan Hu, Hichem Sahli, Xing Fa Dong, Jian Wang, "A High Efficient System For Traffic Mean Speed Estimation from MPEG Video,” In 2009 International Conference on Artificial Intelligence and Computational Intelligence, Beijing, China, pp. 444-448,2009.

[8] Y. Yuan, J. Van Lint, R.E. Wilson, F. van Wageningen-Kessels, S.P.Hoogendoorn, "Real-time lagrangian traffic state estimator for freeways, ” IEEE Trans. Intell. Transp. Syst. pp.59-70, 2012.

[9] S.C. Hsia, P.Y. Hong, "Very large scale integration (VLS) implementation of low-complexity variable block size motion estimation for H.264/AVC coding,” IET Circuits, Devices \& Systems, vol. 4, pp.414-424, 2010.

[10] V. Kastrinaki, M. Zervakis, K. Kalaitzakis, "A survey of video processing techniques for traffic applications," Image and Vision Computing, Vol. 21, pp.359-381, 2005.

[11] B.S. Kerner, Introduction to Modern Traffic Flow Theory and Control, Springer, 2009. 